\title{
IL BOOM DI ACCESSI PER DISTURBI NEUROPSICHIATRICI NEI PRONTO SOCCORSO
}

I risultati di un'indagine condotta dalla Società Italiana di Pediatria in 9 Regioni italiane restituiscono la fotografia di cosa è successo (e di cosa sta succedendo) durante la pandemia.

I primi due casi italiani di Covid- I 9 sono stati confermati il 30 gennaio 2020, quando due turisti provenienti dalla Cina sono risultati positivi al virus SARS-CoV-2 a Roma. Dal 23 febbraio, dopo la scoperta di alcuni focolai, vengono posti in quarantena dieci Comuni nel Nord Italia e, in alcune Regioni, chiuse momentaneamente scuole e università.

Tra l'8 e il 9 marzo la quarantena viene estesa a 26 Province italiane. Dal 5 marzo viene sospesa in tutto il territorio nazionale la didattica in presenza per le scuole di ogni grado e le Università. Con un nuovo DPCM il 9 marzo vengono quindi estesi a tutta Italia il divieto di spostamento per motivi non necessari, la sospensione delle attività sportive, di manifestazioni ed eventi, la chiusura di musei, luoghi di cultura e Centri sportivi.

Ulteriori misure restrittive entrano in vigore con il «Decreto loRestoaCasa», pubblicato l'II marzo, che prevede la sospensione delle comuni attività commerciali al dettaglio, dei servizi di ristorazione, delle celebrazioni religiose, e vieta gli assembramenti di persone in luoghi pubblici o aperti al pubblico.

II 22 marzo una nuova ordinanza, adottata congiuntamente dal ministro della Salute e dal Ministro dell'Interno, vieta a tutte le persone fisiche di spostarsi in qualsiasi Comune diverso da quello in cui si trovano, e viene pubblicata una lista di altre attività non ritenute necessarie, che devono essere sospese. Tutte queste misure vengono più volte prorogate, fino a inizio maggio, allorché le misure restrittive si allentano, consentendo lo spostamento tra Regioni e l'apertura tra l'altro di luoghi di ritrovo per i giovani, quali le sale da ballo e discoteche. Tuttavia, con l'aumento dei contagi, si assiste tra l'altro alla successiva chiusura dei luoghi di ritrovo dei giovani, coprifuoco notturno e didattica a distanza per le superiori, nonché misure restrittive per gli spostamenti. A livello mondiale e nazionale vi è un aggiornamento costante, quotidiano, dei casi di infezione e morti da virus SARS-CoV-2.

Ma c'è un'altra pandemia, inaspettata, subdola che negli ultimi mesi sta colpendo i nostri ragazzi. E di questa pandemia non abbiamo un bollettino giornaliero, ma i numeri che la Società Italiana di Pediatria (SIP) ha raccolto ed esaminato parlano chiari. Non ci troviamo cioè solo a fronteggiare la classica malattia SARS-CoV-2-correlata, con febbre, tosse e insufficienza respiratoria. Subdolamente si è diffusa tra giovani e giovanissimi una patologia della mente, che, per comodità di classificazione, si può genericamente definire patologia neuropsichiatrica (NPI). Restrizioni, incertezza, lockdown hanno contribuito negli ultimi mesi a un incremento di ansia, depressione, ideazione suicidaria, disturbi della condotta alimentare. E la richiesta di aiuto ai nostri Pronto Soccorso si fa sempre più insistente.
E se quindi da un lato il numero di accessi ai Pronto Soccorso italiani è calato rispetto all'epoca pre-Covid, per la paura dei contagi e per il diverso contesto epidemiologico che si è creato, quello per patologia neuropsichiatrica è stato in controtendenza. È questa la fotografia che del resto ci viene restituita da un'analisi della SIP, condotta lungo lo stivale.

Le 9 sezioni regionali che hanno analizzato l'andamento epidemiologico in uno o più Centri ospedalieri confermano un incremento di $+84 \%$ di accesso ai Pronto Soccorso italiani per patologia NPI nel periodo marzo 2020 - marzo 202I rispetto al periodo pre-Covid (marzo 2019 - marzo 2020). Le Regioni in cui si è documentato un maggiore incremento di accessi per patologie NPI sono Emilia-Romagna (+1 10\%), Lazio (+107, $1 \%)$ e Lombardia (+100\%). Nel dettaglio, le patologie per le quali si è osservato un maggiore incremento percentuale degli accessi sono state ideazione suicidaria $(+147 \%)$, depressione (+1 15\%) e disturbi della condotta alimentare $(+78,4 \%)$. Psicosi e disturbi del comportamento alimentare hanno costituito nel campione osservato le prime due cause di accesso in Pronto Soccorso per patologia NPI (rispettivamente 375 accessi su 2242 pari al 16,7\% e 358 accessi pari al 15,9\%). In particolare il maggior incremento di accessi per ideazione suicidaria si è osservato in Lazio ( $+56 \%)$ e Friuli-Venezia Giulia (+204,8\%); il maggior incremento per disturbi della condotta alimentare in Lombardia $(+\mid 74,3)$. I casi di depressione sono stati segnalati soprattutto in Lazio $(+63,4 \%)$. Ma non solo. Posti letto occupati al massimo della loro capienza per settimane e netto innalzarsi delle richieste di aiuto, ovvero un sensibile incremento delle ospedalizzazio$\mathrm{ni}$, che hanno sfiorato il $40 \%$. Anche in questo caso la principale causa è l'ideazione suicidaria (+134\%) seguita da depressione $(+4 I, 4 \%)$ e disturbi della condotta alimentare $(+3 I, 4 \%)$. Questi dati ci restituiscono cioè la fotografia di una patologia in costante aumento tra i nostri ragazzi, sempre più di difficile gestione sul territorio e che richiede pertanto un attento monitoraggio e un rapido intervento capillare da parte delle Istituzioni.

\section{Metodologia dello studio}

Uno o più Centri di riferimento per ciascuna delle Sezioni SIP regionali.

Ricercare retrospettivamente i codici di dimissione dal PS/DEA o, in caso di ricovero dal reparto, compatibili con disturbi mentali, in particolare disturbi psicotici (codifica 29|-299), disturbi nevrotici e altri non psicotici (codifica 300-3I4), ideazione suicidaria (codifica v 62.84) quale diagnosi principale. Totale codici esaminati: 310.

Periodo di riferimento: I marzo 2019-I marzo 202I, da suddividere in 2 sotto periodi $\left(I^{\circ}\right.$ marzo 2019 - $I^{\circ}$ marzo 2020 e 2 marzo 2020 - 2 marzo 202I, rispettivamente denominati periodo pre-Covid e Covid). 

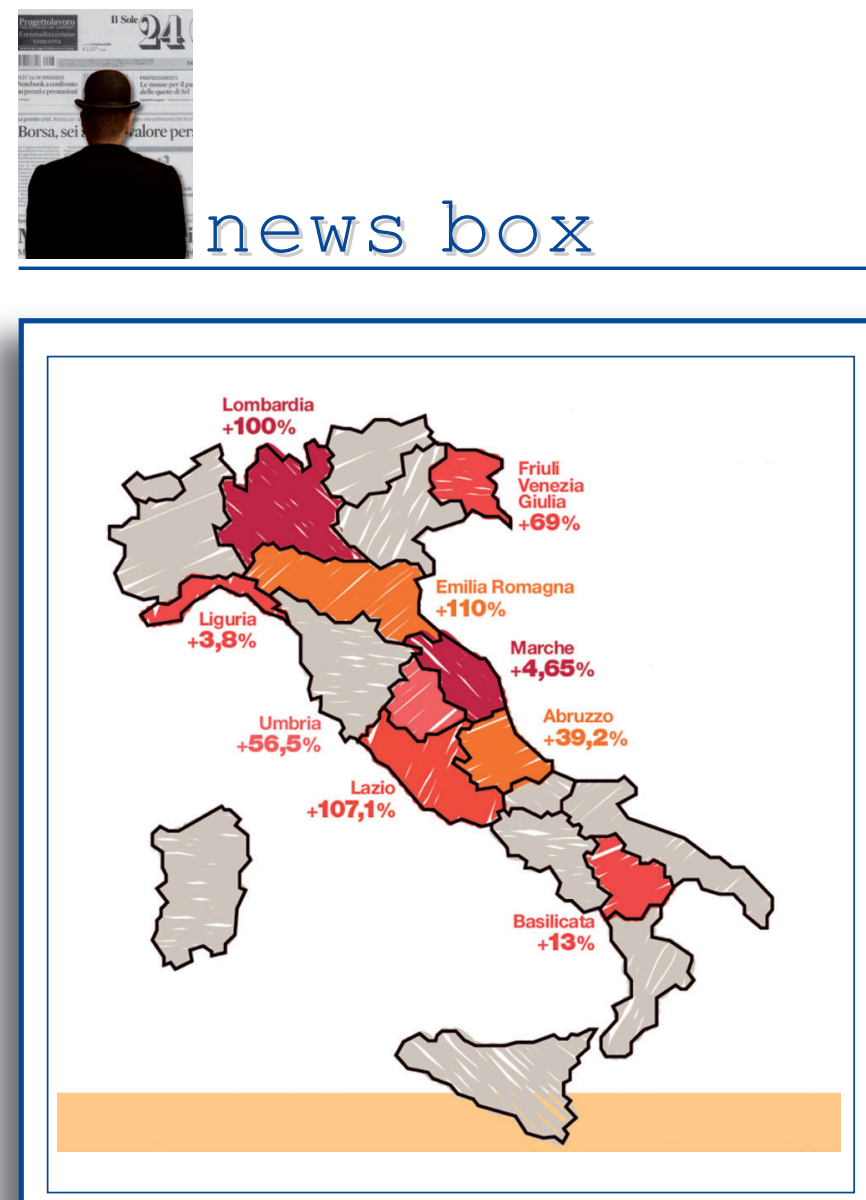

\section{DELLA SOCIETÁ ITALIANA DI PEDIATRIA}

Testo a cura di:

Elena Bozzola, Segretario SIP

Pietro Ferrara, Segretario del GDS SIP Diritti dei Bambini

\section{Centri partecipanti:}

- Abruzzo: Francesco Chiarelli, Federica Cavallo,

Giovanni Farello, Nadia Rossi, Carmela Salladini

- Basilicata: Sergio Manieri, Maria Pia Mirauda

- Emilia-Romagna: Giacomo Biasucci, Andrea Cella,

Gianluca Vergine, Angela Troisi, Federico Marchetti,

Enrico Valletta, Marcello Stella, Marcello Lanari, Duccio Maria

Cordelli, Ilaria Corsini, Jacopo Pruccioli, Chiara Ghizzi,

Chiara Franzonello

- Friuli Venezia Giulia: Egidio Barbi, Alessandro Amaddeo,

Ilaria Liguoro, Paola Cogo, Giuliana Morabito

- Lazio: Pietro Ferrara, Elena Bozzola, Maria Rosaria Marchili, Giulia Spina, Alberto Villani

- Liguria: Riccardo Borea, Emanuela Piccotti, Tommaso Bellini

- Lombardia: Carlo Agostoni, Raffaele Badolato, Camilla Dallavilla

- Marche: Leonardo Felici, Simone Mattozzi

- Umbria: Guido Pennoni, Elisabetta Mencaroni

Tratto e adattato da: Pediatria 2021 ; 9:8-9. 